\title{
Nanoscale electron-beam-driven metamaterial light sources
}

\author{
G. Adamo $^{1}$, K. F. MacDonald ${ }^{1 *}$, F. De Angelis ${ }^{2,3}$, E. Di Fabrizio ${ }^{2,3}$ and N. I. Zheludev ${ }^{1}$ \\ 1. Optoelectronics Research Centre, University of Southampton, Highfield, Southampton, Hampshire, SO17 1BJ, UK \\ 2. Fondazione Istituto Italiano di Tecnologia, NanoBioScience Laboratory, via Morego 30, 16163 Genova, Italy \\ 3. BIONEM Lab, University of Magna Graecia, Campus S. Venuta, Germaneto, viale Europa, 88100 Catanzaro, Italy \\ *kfm@orc.soton.ac.uk; www.nanophotonics.org.uk
}

\begin{abstract}
Free-standing and fiber-coupled photonic metamaterials act as nanoscale, freeelectron-driven, tuneable light sources: emission occurs at wavelengths determined by structural geometry in response to electron-beam excitation of metamaterial resonant plasmonic modes.
\end{abstract}

Considerable effort is currently directed towards the development of nanoscale light (ultimately laser) and surface plasmon ('spaser') sources for a wealth of potential nanophotonic applications. We report here on an experimental demonstration of the fact that planar photonic metamaterials - structured metal films of substantially sub-wavelength thickness - can emit light at wavelengths dictated by their design parameters in response to free-electron excitation.

Our experiments employ planar arrays of asymmetrically-split ring (ASR) resonators - a metamaterial structure that supports a collective trapped-mode plasmonic resonance. These were fabricated by focused ion beam milling through $\sim 70 \mathrm{~nm}$ thick gold films evaporated either on $\mathrm{Si}_{3} \mathrm{~N}_{4}$ membranes or directly onto the end-faces of optical fiber waveguides. A scanning electron microscope provides simultaneously for imaging and targeted electron beam excitation of samples using beam trajectories both perpendicular and parallel to the metamaterial surfaces, as illustrated in Fig. 1.
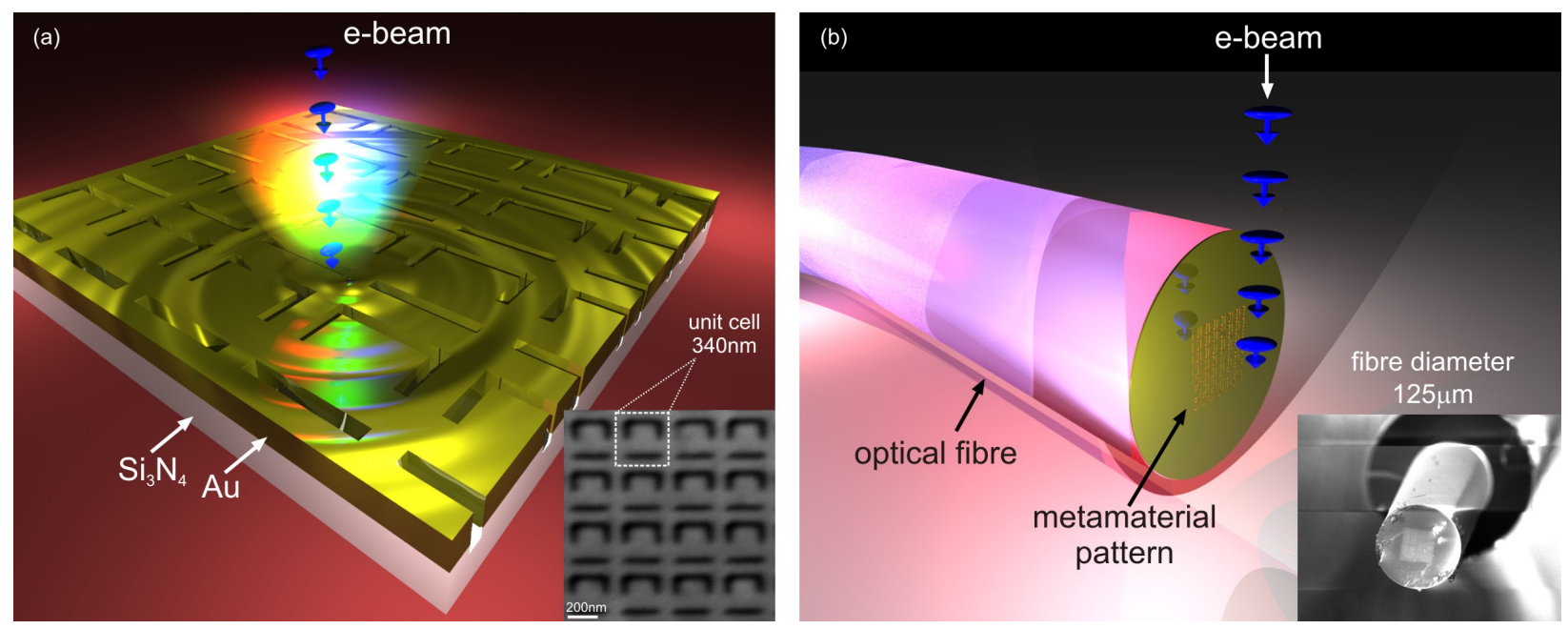

Fig. 1: Artistic impressions of photonic metamaterial light sources driven by free-electron beams. (a) A gold ASR metamaterial on a $\mathrm{Si}_{3} \mathrm{~N}_{4}$ membrane excited by electrons normally incident on the surface; (b) A fibre-coupled gold ASR array excited by an electron beam with a trajectory parallel to the metamaterial surface. The insets show secondary electron images of the corresponding experimental samples. 
In both cases, energy is coupled from incident electrons to the plasmonic modes of the metamaterial structure for which propagating light modes constitute a decay channel. Emitted light is collected for spectroscopic analysis either using a parabolic mirror located directly above the sample, or via the optical fiber substrate.

Fig. 2 compares the electron-induced light emission spectrum of ASR arrays after subtraction of the contribution from unstructured gold (measured at another location on the same sample) with the metamaterials' optical absorption spectra (obtained using a microspectrophotometer). Measurements conducted for a selection of ASR dimensions reveal a direct correlation between the spectral positions of optical absorption peaks and the enhancement of electron-induced metamaterial light emission (relative that of unstructured gold) as well as a red shift in emission wavelength with increasing ASR size. Numerical simulations suggest that the spatial structure of the plasmonic modes excited in the metamaterial dictate the efficiency with which they can couple to light in free space.
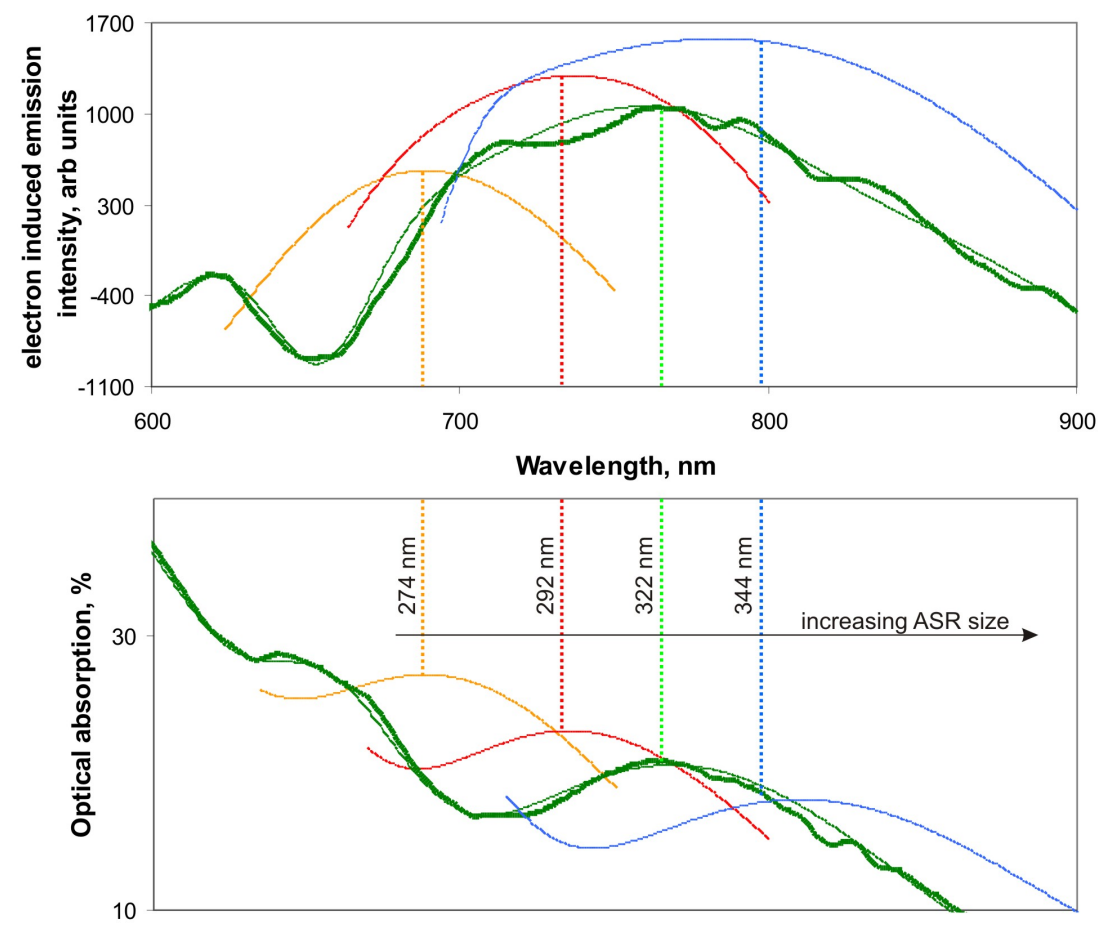

Fig. 2: Electron-induced light emission for gold metamaterial arrays on a $\mathrm{Si}_{3} \mathrm{~N}_{4}$ membrane $[40 \mathrm{keV}$ electrons normally incident on the sample], relative to that from unstructured gold, and corresponding optical absorption spectra. Experimental data and a multiple-Gaussian fitting are shown for an ASR unit cell size of $322 \mathrm{~nm}$ [green lines]; For clarity, truncated fittings only are shown for other unit cell sizes.

In summary, it is demonstrated that a low energy beam of free electrons can excite the collective plasmonic modes of a planar photonic metamaterial array, thereby driving resonant light emission at wavelengths determined by the structural design parameters of the metamaterial, which may be adjusted for operation across the visible to infrared range and beyond. 